\title{
Educação em enfermagem no Brasil e na Bahia: o ontem, o hoje e o amanhã
}

Recebido em: 10/05/2010

Aceito em: 20/04/2011

\author{
Josicelia Dumêt Fernandes \\ Rosana Maria de Oliveira Silva ${ }^{2}$ \\ Lyra Cândida Calhau ${ }^{3}$
}

O presente documento estuda a Educação em Enfermagem (EE) no país e a contribuição das enfermeiras baianas nesse processo. Objetiva registrar a trajetória da EE no Brasil e na Bahia, inserindo-a no contexto do ontem, do hoje e de uma perspectiva do amanhã. Trata-se de um estudo teórico descritivo, tendo como referência estudos dessa área temática. A análise foi realizada por meio de apreciação das relações internas e externas do caminho institucional da $\mathrm{EE}$, resgatando as lutas e conquistas das enfermeiras na construção de um ensino de qualidade. Ficou evidenciado que a lucidez e garra dos sujeitos envolvidos garantiram um espaço político-social que cimenta o presente e viabiliza o futuro da EE com qualidade.

Descritores: Educação em Enfermagem, História da Enfermagem, Enfermagem.

Nursing education in Brazil and in Bahia: yesterday, today and tomorrow

This paper studies the Nursing Education (EE) in the country and the contribution of Bahia nurses in this process. It aims to record the history of EE in Brazil and in Bahia, inserting it in the context of yesterday, today and tomorrow's perspective. This is a theoretical description, with reference to studies of this subject area. The analysis was performed by assessing the internal and external relations of the path of institutional EE, rescuing the struggles and achievements of nurses in the construction of a quality education. It was evident that the clarity and vigor of the individuals involved have ensured political and social space that cements the present and enables the future of EE with quality.

Descriptors: Nursing Education, Nursing History, Nursing.

\section{Educación en enfermería en Brasil y en Bahía: ayer, hoy y mañana}

Este trabajo estudia la Educación en Enfermería (EE) en el país y la contribución de las enfermeras de Bahía en este proceso. Su objetivo es registrar la historia de la EE en Brasil y en Bahía, insertándola en el contexto de ayer, hoy y en la perspectiva de mañana. Se trata de una descripción teórica, con referencia a los estudios de este asunto. El análisis se realizó mediante la evaluación de las relaciones internas y externas de la trayectoria institucional de la $\mathrm{EE}$, el rescate de las luchas y los logros de las enfermeras en la construcción de una educación de calidad. Es evidente que la lucidez y el vigor de los individuos implicados han asegurado un espacio político y social que consolida el presente y permite el futuro de la EE con calidad.

Descriptores: Educación en Enfermería, Historia de la Enfermería, Enfermería.

\section{INTRODUÇÃO}

$\mathrm{F}$ rente às tendências pedagógicas na área da educação, este estudo buscou a construção de um conhecimento sobre a Educação em Enfermagem (EE) no país e a contribuição das enfermeiras baianas nesse processo, partindo do entendimento de que a compreensão da realidade da $E E$, sua trajetória, tendências e contradições são fatores decisivos para a compreensão do hoje e o direcionamento do amanhã. Buscou-se, portanto, a configuração de um passado e o reconhecimento de possibilidades presentes e futuras.

Sob esse entendimento, objetivou-se registrar a trajetória da $\mathrm{EE}$, no Brasil e na Bahia, inserindo-a no contexto do ontem, do hoje e de uma perspectiva do amanhã.

Trata-se de um estudo teórico descritivo, tendo como referência estudosdessaáreatemática.Aanálisefoirealizadaatravésdaapreciação do ontem, do hoje e de uma perspectiva do amanhã, evidenciando- se as relações internas e externas do caminho institucional da EE no Brasil e na Bahia, resgatando as lutas e conquistas das enfermeiras na construção de um ensino de qualidade.

\section{O ONTEM}

Emergência do ensino de enfermagem no Brasil e na Bahia No Brasil, a primeira tentativa de sistematização do ensino de enfermagem ocorreu em 1890, quando da criação, na cidade do Rio de Janeiro, da Escola Profissional de Enfermeiros, no Hospício Nacional de Alienados, com a finalidade de preparar enfermeiros para trabalhar em hospícios e hospitais civis e militares ${ }^{(1-2)}$.

Por volta de 1901, foi criado outro curso, no então Hospital Evangélico, em São Paulo, com o objetivo de preparar pessoal para o referido hospital, que se destinava ao atendimento de estrangeiros residentes no Brasil $^{(1-2)}$.

1 Enfermeira. Professora titular da Escola de Enfermagem da Universidade Federal da Bahia-UFBA. Líder do Grupo de Estudos e Pesquisas em Educação, Ética e Exercício de Enfermagem da Universidade Federal da Bahia - Exerce. Pesquisadora do CNPq. E-mail: dumet@ufba.br.

2 Enfermeira. Professora adjunta da Escola de Enfermagem da UFBA. Doutoranda do programa de pós-graduação em enfermagem da UFBA. Membro do Grupo de Estudos Exerce.

3 Enfermeira. Professora da graduação em enfermagem da Universidade do Sudoeste da Bahia. Doutoranda do programa de pós-graduação em enfermagem da UFBA.

Membro do Grupo de Estudos Exerce. 
A Cruz Vermelha Brasileira deu início, por volta de 1916, na cidade do Rio de Janeiro, à Escola Prática de Enfermeiras da Cruz Vermelha, com o propósito de preparar voluntários para as emergências de guerra ${ }^{(1-2)}$.

Esses cursos, criados para atender às necessidades emergenciais de cada momento histórico, não atendiam aos padrões da enfermagem moderna, que, em 1860, já haviam sido estabelecidos em Londres por Florence Nightingale. Atribui-se a isso o fato de que, naquela época, não havia, por parte do Estado, uma política explícita que indicasse uma preocupação com a saúde da população.

A problemática da saúde pública, na década de 1920, veio demandarnovaseamplas respostas doEstado, ese destacama criação do Departamento Nacional de Saúde Pública e, posteriormente, em 1923, da Escola de Enfermeiras desse Departamento. Mais tarde, essa escola passou a chamar-se Escola de Enfermeiras D. Ana Néri, em homenagem à primeira enfermeira brasileira, a baiana Anna Justina Ferreira Nery, que serviu como voluntária na Guerra do Paraguai ${ }^{(3)}$.

Nascida no município de Cachoeira de Paraguaçu-BA, ao ver seus filhos e irmãos serem convocados para a Guerra do Paraguai, escreveu uma carta ao então presidente da província, oferecendo seus serviços como enfermeira. Partiu da Bahia em 1865 e começou seu trabalho no hospital de Corrientes. Com os próprios recursos, montou, na capital conquistada, na casa onde morava, uma enfermaria onde trabalhou abnegadamente. De volta ao Brasil, em 1870, foi condecorada com as medalhas de prata humanitária e da campanha e recebeu do imperador uma pensão vitalícia.

A Escola de Enfermeiras D. Ana Néri, criada dentro dos moldes nightingalianos, nasceu na época áurea da saúde pública e teve como objetivo explícito a melhoria das condições sanitárias da população.

A emergência do ensino de enfermagem moderna em nosso país coincidiu, pois, com o momento em que surgiam os primeiros traços de uma política de saúde por parte do Estado, ou seja, com o momento em que a questão da saúde ganhava uma nova dimensão, tornando-se uma das atribuições do Estado.

O segundo pós-guerra, a aceleração do processo de substituição das importações e o fortalecimento do processo de industrialização, a partir da década de 1940, evidenciaram a necessidade de uma força de trabalho qualificada e saudável, o que implicava na necessidade de profissionais qualificados.

Nesse contexto, foi criada a Escola de Enfermagem da Bahia (EEUB), em janeiro de 1946. Começando a funcionar no ano seguinte, foi incorporada à Universidade da Bahia. Mais tarde, em 1968, com a reestruturação da Universidade Federal da Bahia, essa Escola converteu-se em uma Unidade de Ensino Profissional e Pesquisa Aplicada, de nível superior ${ }^{(4)}$.

O então reitor da Universidade da Bahia, doutor Edgar Santos, convidou para dirigir essa escola a enfermeira baiana dona Haydée Guanais Dourado, que possuía o perfil necessário para o desempenho da honrosa e difícil incumbência recebida.
Dona Haydée era graduada em enfermagem pela Escola Anna Nery; especialista em pedagogia, didática e administração de enfermagem pela Universidade de Toronto/Canadá; bacharel em ciências políticas e sociais pela Escola de Sociologia e Política da Universidade de São Paulo; membro do corpo docente da Escola de Enfermagem da Universidade de São Paulo (USP). Sócia efetiva da Associação Brasileira de Enfermagem (ABEn) desde 1944, ela participou da entidade com destaque na organização dos congressos, na expansão das seções, na luta por uma legislação para a categoria, na realização do estudo das necessidades de enfermagem no país, entre inúmeras outras. Conquistou amplos espaços para a enfermagem baiana, vencendo muitas barreiras ao projetar essa escola em altos níveis. Criou os alicerces do ensino da enfermagem baiana.

\section{A institucionalização do ensino de enfermagem}

O Estado, atendendo à lógica de produção de serviços de saúde e através da Lei n 775 de 06/08/1949, propôs a ampliação do número de escolas, tornando obrigatória a existência do ensino deenfermagemem todocentrouniversitário ou sede de faculdades de medicina, além de definir um ensino voltado para a área hospitalar, centrado no modelo clínico e com aderência ao mercado de trabalho à época ${ }^{(1-2)}$.

O ensino de enfermagem no Brasil, destarte, apesar de ter sido criado em 1923, sob a influência do sanitarismo, só a partir do desenvolvimento industrial e da modernização dos hospitais é que passou a ser consolidado como matéria de lei. A institucionalização da EE no Brasil, portanto, está contida num contexto que ganha suas amplas dimensões quando remetida aos problemas de organização e funcionamento da sociedade e do Estado.

Essa lei, contudo, trouxe algumas insatisfações às educadoras de enfermagem que, reunidas durante o IV Congresso Nacional de Enfermeiras, realizado em Salvador, em 1950, recomendaram "a necessidade de acentuar-se o ponto de vista das medidas preventivas em saúde, em todas as matérias do curso"(4).

O Estado, se por um lado reconheceu o ensino de enfermagem como matéria de lei, em resposta às pressões exercidas pelas enfermeiras, por outro, atendendo ao setor produtivo e empresarial da área da saúde, definiu a obrigatoriedade do ensino das ciências físicas e biológicas, sendo limitativo no que se referia às ciências sociais ${ }^{(1,2,4)}$.

Em meio às discussões daquela lei, foi realizado em Salvador, em 1958, por solicitação da ABEn, com apoio da Universidade da Bahia, o "Seminário Didático Internacional sobre Levantamentos de Enfermagem", patrocinado pela Organização Mundial de Saúde. A escolha do local recaiu na cidade de Salvador"por contar a cidade com uma das escolas de enfermagem mais reputadas do país e pelo conceito que desfruta essa especialidade nos meios universitários do Estado, o que torna o ambiente sobremodo propício à efetivação de estudos internacionais"(5). 


\section{Um currículo mínimo para o curso de enfermagem}

Em 1961, com a Lei 4024, que fixou as Diretrizes e Bases da Educação Nacional, o currículo mínimo para os cursos de graduação em enfermagem passou a ser fixado através do Parecer no $271 / 62^{(6)}$, que estabeleceu um curso geral e duas alternativas para especialização precoce, além de reduzir a duração de quatro para três anos e excluir as disciplinas de saúde pública e ciências sociais.

A ABEn, através do Ofício $n^{\circ} 115$ de $27 / 10 / 62^{(7)}$, solicitou ao então Conselho Federal de Educação (CFE) que sustasse a homologação daquele Parecer, ao mesmo tempo que propôs alterações substanciais. Essas propostas, contudo, não obtiveram eco, sendo mantidas, em essência, as distorções do novo currículo adotado: concepção do social diluída em função da concepção exclusiva do biológico, com o ensino voltado para o atendimento ao doente hospitalizado e com o predomínio das medidas curativas em saúde.

Procurando atenuar as insatisfações dos profissionais de enfermagem com o Parecer 271/62, o Estado elevou o ensino de enfermagem à condição de nível superior. $\mathrm{A}$ partir de então, observa-se maior demanda de candidatos para os cursos de enfermagem. Entretanto, se por um lado houve maior demanda por esses cursos, por outro, ocorreu o fechamento de algumas escolas que não estavam em condições de ter seus cursos elevados a nível superior ${ }^{(1)}$.

A EEUB, através de suas docentes e discentes, participou ativamente, sob a coordenação da ABEn, dos esforços para reverter as distorções daquele Parecer. Nesse movimento, destaca-se a atuação da professora Maria Ivete Ribeiro de Oliveira, que dirigiu a escola entre 1963 a 1966 (primeiro período) e 1966 a 1970 (segundo período). Nascida na Bahia, graduada em enfermagem pela Universidade da Bahia e em filosofia pela Universidade Católica de Salvador, pós-graduada na Universidade de Boston e de Los Angeles e livre-docente pela USP, ocupou cargos de adjunto de reitor para assuntos de ensino, pesquisa e extensão da UFBA. Foi presidente da ABEn e do Cofen. Teve papel vital no desenvolvimento da enfermagem na Bahia e no Brasil, lutando, em níveis nacional e internacional, para que a enfermagem se desenvolvesse e assumisse novos significados na sociedade.

Vale destacar que, em 1964, no XVI Congresso Brasileiro de Enfermagem, sediado em Salvador, o tema "Enfermagem e Pesquisa"foi, pela primeira vez, abordado como tema central em um congresso de enfermagem. A Bahia desencadeou essa discussão capitaneada pela doutora Maria Ivete Ribeiro de Oliveira.

\section{A Reforma Universitária}

Em 1968, a Lei no 55401, Lei da Reforma Universitária (RU)*, fixou as normas de organização e funcionamento do ensino superior, além de definir a ampliação do número de vagas, a modernização do ensino superior e a necessidade de revisão dos currículos mínimos dos cursos. Atendia, também, às exigências de um mercado de trabalho que requeria um número crescente de profissionais qualificados ${ }^{(1-2)}$.

Assim, em resposta às determinações do então CFE, as docentes de enfermagem passaram a movimentar-se através de uma série de eventos, no sentido de formular uma proposta de currículo e efetivar as diretrizes emanadas da $\mathrm{RU}^{(8)}$.

Essa proposta foi transformada em anteprojeto do currículo mínimo eenviada, através da ABEn, ao então CFE. Após sofrer modificações, ela converteu-se no Parecer n 163/72 da Comissão Central de Revisão de Currículos, aprovado pela Resolução CFE n4 de 25/2/72, que definiu o currículo mínimo para os cursos de graduação em enfermagem.

O novo currículo atendeu, em parte, às pressões das profissionais de enfermagem, pois possibilitou o aumento da duração do curso e a inclusão do ensino das ciências sociais. No entanto, ele não trouxe grandes transformações, pois manteve o modelo biologicista, individualista e hospitalocêntrico, além de uma visão tecnicista da saúde ${ }^{(1,2)}$.

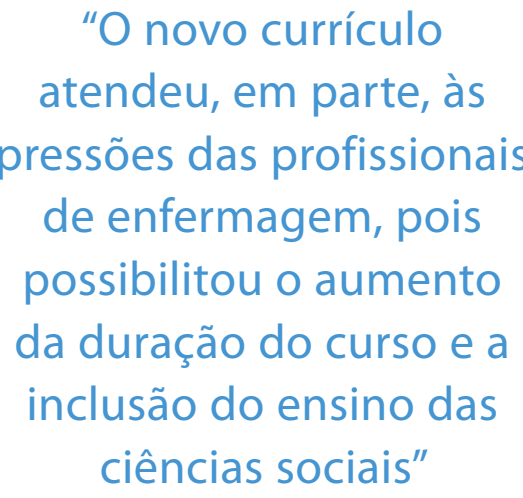

O início da pós-graduação em enfermagem No Brasil, a pós-graduação foi instituída pela Lei de Diretrizes e Bases da Educação Nacional, em 1961, e aprovada pelo CFE, em 1965, através do Parecer 977. Após essa lei, a RU institucionalizou a pós-graduação stricto sensu, objetivando formar pessoal qualificado para ampliar o quadro docente e estimular estudos e pesquisas para o desenvolvimento do país ${ }^{(1,2)}$

$\mathrm{Na}$ área da enfermagem, a pós-graduação stricto sensu teve seu início em 1972, com a criação do curso de mestrado da Escola de

Enfermagem da Universidade Federal do Rio de Janeiro e, em 1981, com o primeiro curso de doutorado do Programa Interunidades de Doutorado em Enfermagem, envolvendo a Escola de Enfermagem da USP e a Escola de Enfermagem de Ribeirão Preto da USP.

Na Bahia, a pós-graduação stricto sensu foi criada em 1978 e iniciou seu funcionamento em 1979, com seu curso de mestrado em enfermagem com área de concentração em enfermagem médicocirúrgica; mais tarde, considerando a demanda da comunidade, foram criadas as áreas de atenção à saúde da mulher e da criança e de administração de serviços de enfermagem. Esse curso veio sanar a carência da Região Nordeste, respondendo às necessidades de capacitação para a docência, assistência e pesquisa ${ }^{(9)}$.

\section{Mais um currículo para o curso de graduação em enfermagem} $\mathrm{Na}$ década de 1980, vinculados aos movimentos sociais de redemocratização brasileira, surgiram novas propostas de saúde, em que os princípios da equidade, integralidade e universalidade passaram a nortear as políticas do setor, com o Sistema Único de Saúde (SUS).

A ABEn, como um dos atores engajados nesse processo de luta, desencadeou um amplo debate, mobilizando docentes, discentes

* Com a RU, em 1968, a Universidade Federal da Bahia foi reestruturada e a EEUB tornou-se Unidade de Ensino Profissional e Pesquisa Aplicada, passando a ser denominada Escola de Enfermagem da Universidade Federal da Bahia (EEUFBA). 
e profissionais dos serviços, objetivando a construção coletiva de um projeto educacional para a enfermagem brasileira.

A Escola de Enfermagem da Universidade Federal da Bahia (EEUFBA) participou ativamente desses debates, tendo uma docente atuando na Comissão de Educação da ABEn.

A troca de experiências e a riqueza das discussões geradas nesse movimento subsidiaram o Parecer 314/94 do então CFE, homologado pela Portaria $1721 / 94^{(10)}$ do Ministério da Educação, surgindo, assim, mais um novo currículo para os cursos de graduação em enfermagem. Constituído por quatro eixos temáticos, a saber: bases biológicas e sociais da enfermagem; fundamentos de enfermagem; assistência de enfermagem; e administração em enfermagem.

Além de determinar os eixos temáticos e o percentual da carga horária dos mesmos, a Portaria 1721/94 definiu, também, a carga horária mínima do curso em 3.500 horas e sua duração em, no mínimo, quatro anos (ou oito semestres letivos) e, no máximo, seis anos (12 semestres letivos).

Esse novo currículo, apesar de contemplar algumas propostas apresentadas pelas profissionais de enfermagem, gerou algumas insatisfações, tais como: ausência das disciplinas da área de educação,desconsiderandoafunçãoeducativa da enfermagem; delimitação do percentual da carga horária dos eixos temáticos, o que configurava um controle sobre o processo de formação, desconsiderando as características institucionais e regionais de cada curso.

Essas insatisfações eram manifestadas nos diversos eventos científicos da área, particularmente nos Seminários Nacionais de Diretrizes para a Educação em Enfermagem (Senadens), criados pela ABEn a partir de 1994 e que trouxeram contribuições significativas para a construção das Diretrizes Curriculares Nacionais do curso de graduação em enfermagem.

\section{Diretrizes Curriculares Nacionais do curso de graduação em enfermagem}

Em 1996, foi aprovada a LDB, Lei 9394 de 20/12/96 (11) , que abriu espaços para a flexibilização dos currículos, para a expansão de cursos/vagas na educação superior, além de direcionar a construção de DCN.

Após esse parecer, foi aprovada a Resolução CNE/CES no 03 de $7 / 11 / 2001^{(12)}$, que definiu as DCN.

Essas diretrizes trazem as competências gerais, específicas e habilidades pautadas nas concepções do aluno como sujeito de seu processo de formação, articulação entre teoria e prática, diversificação dos cenários de aprendizagem, metodologias ativas, articulação da pesquisa com o ensino e extensão, flexibilidade curricular, interdisciplinaridade, incorporação de atividades complementares, avaliação da aprendizagem, processo de acompanhamento, avaliação e gestão do curso, assim como da terminalidade do curso ${ }^{(13)}$.

Essas diretrizes tiveram sua materialidade concretizada a partir de propostas que emergiram da mobilização das(os) enfermeiras(os), através da $A B E n$, de entidades educacionais e de setores da sociedade civil interessados nas mudanças da formação na área da saúde. Elas expressam a necessidade do compromisso com princípios da Reforma Sanitária Brasileira e com o Sistema Único de Saúde (SUS).

Na elaboração das DCN, a Bahia teve um papel de fundamental importância através da Carta da Bahia que, juntamente com a Carta de Natal, deram subsídios para a Carta de Florianópolis, originária da plenária da $\mathrm{EE}$ realizada durante o $51^{\circ}$ Congresso Brasileiro de Enfermagem, em 1999, em Florianópolis.

Vale destacar o papel da Comissão de Especialistas de Ensino de Enfermagem CEEEnf da Secretaria de Ensino Superior do MEC, que, acatando a Carta de Florianópolis, mobilizou-se no sentido de garantir a aprovação, pelo Conselho Nacional de Educação, das propostas emanadas da categoria de enfermagem contidas nas DCENF ${ }^{(14)}$.

A CEEEnf estava constituída de quatro docentes, sendo que uma delas era professora da EEUFBA. Ou seja, a EEUFBA teve intensa participação na elaboração e aprovação das DCENF.

\section{Muitos desafios estão sendo encontrados, tais como: sair do polo de ensino centrado no da aprendizagem no aluno"}

\section{OHOJE}

No momento, as escolas/cursos encontramse em fase de adequação de seus projetos pedagógicos e matrizes curriculares às DCENF. Nesse processo, muitos desafios estão sendo encontrados, tais como: sair do polo de ensino centrado no professor para o polo da aprendizagem centrada no aluno; sair da fragmentação do modelo disciplinar para a construção de um currículo integrado, onde o eixo da formação passa a ser a prática/trabalho/cuidado; sair da teoria antecedendo a prática para a articulação teoria/prática; sair das concepções de saúde como ausência de doença para a saúde enquanto condições de vida; sair da polarização individual/coletivo e biológico/social para uma consideração de interpenetração e transversalidade; sair da concepção de avaliação como processo punitivo para a de avaliação como instrumento de (re)definição de paradigmas ${ }^{(13)}$.

No enfrentamento desses desafios, as escolas/cursos estão sendo estimuladas a definir novas ações de superação, a saber: redirecionar, coletivamente, seus projetos pedagógicos, tendo como paradigma as relações entre cultura, sociedade, saúde e educação; indicar a essencialidade do aluno como sujeito de seu processo de formação; fortalecer a articulação entre teoria e prática, numa contínua aproximação do mundo do ensino com o mundo do trabalho; utilizar metodologias ativas que possibilitem aos estudantes ocupar o lugar de sujeitos na construção de sua aprendizagem e que colocam o professor como facilitador e orientador desse processo; aprofundar a articulação da pesquisa com o ensino e a extensão, favorecendo o desenvolvimento da capacidade de produzir conhecimento próprio e inovador; incrementar a flexibilidade curricular, evitando a rigidez dos pré-requisitos e de conteúdos obrigatórios; integrar diferentes campos de conhecimento, possibilitando uma visão global da realidade e admitindo a ótica pluralista das concepções de ensino, adotando o princípio da interdisciplinaridade como 
forma de superar o pensar simplificado e fragmentado da realidade; incorporar atividades complementares no processo de formação, criando mecanismos de aproveitamento de conhecimentos adquiridos através de estudos e práticas independentes, presenciais e a distância; pôr em prática a avaliação da aprendizagem como um processo formativo e permanente de reconhecimento de saberes, competências, habilidades e atitudes, deixando de ser pontual, punitiva e discriminatória para constituir-se numa avaliação que respeite a individualidade do aluno e que garanta sua formação com qualidade e competência ${ }^{(13)}$.

\section{O AMANHÃ}

A política de globalização do mercado está a exigir, para o amanhã, maior competitividade, frente a um mercado cada vez mais heterogêneo e fragmentado, o que implica na formação de um novo perfil para o enfermeiro: um indivíduo capaz de conhecer e intervir sobre os problemas/situações de saúde-doença mais prevalentes no perfil epidemiológico nacional, com ênfase em sua região de atuação; um indivíduo crítico, reflexivo, capaz de atuar, com senso de responsabilidade social e compromisso com a cidadania, como promotor da saúde integral do ser humano, buscando projetar o novo, para agir com criatividade no enfrentamento das dificuldades do mercado.

Nesse direcionamento, faz-se necessária a formação de profissionais ativos e aptos a aprender e a atender as tendências do mundo globalizado, garantindo a capacitação de profissionais com autonomia e discernimento para assegurar a integralidade da atenção à saúde e a qualidade e humanização do atendimento prestado aos indivíduos, famílias e comunidades.

O processo de formação deverá, pois, estar comprometido e direcionado à concretização dos princípios da reforma sanitária e do SUS e avançar não apenas no preparo de um novo profissional, mas, também, de um indivíduo crítico, cidadão, preparado para aprender, criar, propor e construir.

\section{CONSIDERAÇÕES FINAIS}

Da análise realizada, percebemos a EE como uma realidade humana sujeita a transformações pela influência da sociedade onde está inserida e pelo resultado da interação das pessoas que a integraram/integram e lhe deram/dão vida. Percebemos, também, que a mudança das estruturas e as relações sociais permitem a configuração de um passado e o reconhecimento de possibilidades presentes e futuras.

A EE deve ser apreendida, portanto, como produto de uma construção social e histórica, constituindo-se numa totalidade organizada, complexa, sujeita a determinações, contendo contradições dialeticamente superadas e em constante mutação. Deve estar fundamentada nas relações dialéticas entre as transformações sociais e as do mundo do trabalho, trazendo como elemento nuclear a integração entre conteúdos teóricos e práticos, competências e habilidades, além de se pautar na produção de conhecimentos inovadores mediante a inserção em realidades concretas, ou seja, a formação centrada na práxis.

As mudanças e os desafios a serem superados no processo de formação dos enfermeiros indicam, pois, a necessidade de redirecionamento dos conteúdos de ensino e da pesquisa, tomando a extensão o eixo articulador. Apontam, assim, para o fortalecimento da qualidade do processo de formação, tendo o SUS como eixo fundante desse processo, configurando o amanhã da educação em enfermagem.

\section{Referências}

1. Fernandes JD. Expansão do ensino de enfermagem no Brasil [tese]. Salvador:

Escola de Enfermagem da Universidade Federal da Bahia; 1998.

2. Fernandes JD. Trajetória do ensino de graduação em enfermagem no Brasil. In:

Teixeira E, Vale EG, Fernandes JD, Sordi MRL, organizadores. O ensino de graduação

em enfermagem no Brasil: o ontem, o hoje e o amanhã. Brasília: MEC/Inep; 2007.

3. Ministério da Saúde (BR). Fundação Serviços de Saúde Pública. Enfermagem:

legislação e assuntos correlatos. 3a ed. Rio de Janeiro: GB/Fsesp; 1974.

4. Oliveira MIR. Emergência e inserção da Escola de Enfermagem na comunidade

acadêmica da Universidade da Bahia. In: Fernandes JD, organizador. Memorial Escola

de Enfermagem 1946-1996. Salvador: UFBA; 2001.

5. Oliveira MIR. A consolidação da Escola de Enfermagem da Universidade da Bahia.

In: Fernandes JD, organizador. Memorial Escola de Enfermagem 1946-1996. Salvador: UFBA; 2001.

6. Conselho Federal de Educação (BR). Parecer n 271 de 19 de outubro de 1962.

Dispōe sobre o currículo mínimo do curso de enfermagem. In: Ministério da Saúde

(BR). Fundação Serviços de Saúde Pública. Enfermagem, legislação e assuntos

correlatos. 3a ed. Rio de Janeiro: Ministério da Saúde; 1974. p. 249-53.

7. Associação Brasileira de Enfermagem. Ofício no 115 de 27 de outubro de 1962. Rev Bras Enferm. 1963;16(1):14-6.

8. Oliveira MIR. A reforma universitária e o Curso de Enfermagem da Universidade da
Bahia. In: Fernandes JD, organizador. Memorial Escola de Enfermagem 1946-1996. Salvador: UFBA; 2001.

9. Fernandes JD. O fortalecimento das atividades acadêmicas da Escola de Enfermagem da Universidade Federal da Bahia, 1979 a 1989. In: Fernandes JD, organizador. Memorial Escola de Enfermagem 1946-1996. Salvador: UFBA; 2001. 10. Ministério da Educação (BR). Portaria no 1721 de 16 de dezembro de 1994: currículo mínimo do curso de enfermagem. Brasília: Diário Oficial da União; 1994. dez 16; Seção 1:19301-2.

11. Ministério da Educação (BR). Lei no 9394 em 20 de dezembro de 1996: estabelece as diretrizes e bases da educação nacional. Brasília: Diário Oficial da União; 199. dez 23; 34 (248); Seção 1:27. 833-41.

12. Ministério da Educação (BR). Conselho Nacional de Educação. Câmara de Educação Superior. Resolução CNE/CES nº 3 de 7/11/2001: Diretrizes Curriculares Nacionais do Curso de Graduação em Enfermagem. Brasilia: Ministério da Educação; 2001. 13. Fernandes JD, Ferreira SLA, Oliva DSR, Santos MP, Costa HOG. Diretrizes estratégias para a implantação de uma nova proposta pedagógica na Escola de Enfermagem da Universidade Federal da Bahia. Rev Bras Enferm. 2003;56(4):392-5. 14. Fernandes JD, Xavier IM, Ceribelli IPF, Bianco MHC, Rodrigues MV, Maeda D. Diretrizes curriculares e estratégias para implantação de uma nova proposta pedagógica. Rev Esc Enferm USP. 2005;39(4):443-9. 